\title{
An examination of diurnal variations in neuropathic pain and affect, on exercise and non-exercise days, in adults with spinal cord injury
}

\author{
Kendra R. Todd ${ }^{1,2} \cdot$ Kathleen A. Martin Ginis $\mathbb{1}^{1,2,3}$
}

Received: 12 June 2018 / Revised: 5 October 2018 / Accepted: 6 October 2018

(c) International Spinal Cord Society 2018

\begin{abstract}
Study design Case series

Objectives The temporal relationships between exercise, neuropathic pain and affect are not well understood. The purpose of this study was to utilize ecological momentary assessment to measure intra-individual diurnal variations in neuropathic pain and affect on exercise and non-exercise days. This study aimed to provide a deeper understanding of how neuropathic pain and affect change from pre- to post-exercise, and over time.

Setting Community.

Methods Six physically active men with SCI participated in a 6-day protocol $\left(M_{\text {age }}=39.33 \pm 8.24 ; 83.3 \%\right.$ tetraplegics; years post injury $=6-17$ years). Using their Smartphones, participants completed the Feeling Scale, Felt Arousal Scale, and Neuropathic Pain Scale in response to six daily prompts, and before and after exercise. Paired samples $t$-tests were conducted on changes in neuropathic pain and affect from pre to post-exercise. Bivariate Pearson's correlational analyses were computed between time of day, neuropathic pain and affect.

Results Participants experienced a significant decrease in neuropathic pain $(t(5)=3.93 ; p=0.01)$ following completion of at least one bout of exercise. A large, but non-significant increase $\left(\mathrm{Hg}_{\mathrm{av}}=0.76\right)$ in Feeling Scale scores occurred following one bout of exercise. Time of day, neuropathic pain and affect were significantly correlated for two participants.

Conclusions Overall, results suggest exercise can reduce neuropathic pain, and may also increase feelings of pleasure. Given the inconsistent pattern of results across participants, further research is needed to look at both individual characteristics, and characteristics of exercise that may moderate changes in neuropathic pain and affect for adults with SCI.
\end{abstract}

\section{Introduction}

Approximately 50\% of individuals with a spinal cord injury (SCI) experience neuropathic pain [1,2]. Neuropathic pain is defined as pain caused by a lesion or disease of the somatosensory nervous system [3] and is considered one of the most debilitating forms of pain $[4,5]$. It commonly

Kendra R. Todd

ktodd03@mail.ubc.ca

1 School of Health and Exercise Sciences, University of British Columbia, Kelowna, BC, Canada

2 International Collaboration on Repair Discoveries (iCORD), Blusson Spinal Cord Centre (BSCC), University of British Columbia, Vancouver, BC, Canada

3 Southern Medical Program, Faculty of Medicine, University of British Columbia, Kelowna, BC, Canada manifests as allodynia [6] (pain resulting from a nonnoxious stimulus) and hyperalgesia (heightened response from a noxious stimulus) [7]. Further, neuropathic pain is often refractory to pharmacotherapy, which is the most frequently prescribed management option $[2,8]$.

Exercise has been proposed as a potential treatment for SCI-related neuropathic pain, with preliminary data supporting its effectiveness [8,9]. In a survey study of 471 adults with SCI, $78 \%$ of those who used exercise for pain management, rated it to be 'extremely helpful' [10]. Additionally, a 7-week treadmill exercise training program was shown to lead to a full elimination of allodynia in rats [11]. Although some studies have examined the effects of exercise on musculoskeletal pain [12-15], to the best of our knowledge, only one study has tested the effects of exercise on neuropathic pain in humans with SCI [16]. This 10-week exercise training study consisted of 3 workouts per week using a seated, double-poling arm ergometer. Study participants experienced a 2-point median reduction in pain 
intensity, with 6 of 10 bodily pain locations decreasing by 1.8 clinically significant units. These results are similar to the magnitude of reduction in neuropathic pain from pharmaceuticals [8]. Importantly, no participants reported an increase in their neuropathic pain resulting from exercise participation.

In addition to the limited research on how neuropathic pain responds to exercise, knowledge is also limited regarding how neuropathic pain fluctuates over the course of a day, and within a real-world context. Neuropathic pain reported in an experimental setting may not accurately represent an individual's typical pain response to exercise and other daily activities [17]. Thus, it is important to measure neuropathic pain in real-world settings. Although one previous SCI research study suggests that pain may exhibit temporal patterns [18], this study did not specifically measure neuropathic pain. One way to address these limitations is to conduct research using ecological momentary assessment (EMA).

EMA involves repeated sampling of participants' behaviours and experiences in real time and within their natural environments [19]. EMA methodology can overcome retrospective recall biases by capturing present pain experiences, increasing the ecological validity of assessments by capturing sensations in a participant's natural social context $[19,20]$. EMA has been used previously in SCI research to observe the momentary associations between chronic pain, pain interference, and physical activity [18], but it has not yet been used to examine neuropathic pain. EMA was used in the present study to examine individual differences in neuropathic pain both in response to exercise, and over the course of a day in adults with SCI. EMA also allowed us to examine study participants' affect in relation to pain and exercise. In both adults with and without SCI, those who experience greater pain have been shown to report more negative affect (i.e., instantaneous displeasure that lacks cognitive appraisal) [21] and mood states (e.g., depression, anxiety) [22-26]. Importantly, individual bouts of exercise have been shown to improve affect in adults with SCI [26].

In summary, the primary purpose of this study was to measure study participants' neuropathic pain over a 6-day observational period and in response to naturalistic exercise. Based on the results of Norrbrink and colleagues' exercise intervention study, it was hypothesized that study participants would report an acute reduction in neuropathic pain immediately following a bout of exercise [16]. A secondary purpose was to examine the relationship between neuropathic pain, affect and exercise. It was hypothesized that overall, fluctuations in affect would mirror fluctuations in pain, and affect would become more positive following exercise [26]. Given the absence of research regarding diurnal variations in neuropathic pain and affect in individuals with SCI, we tested the null hypothesis that there would be no relationship between time of day, neuropathic pain and affect.

\section{Methods}

\section{Participants}

Published case series of individuals with SCI typically include 5-10 cases [27-31]. For this case series, we aimed to recruit individuals with SCI who: were greater than 1 year post injury; experienced below-level neuropathic pain; were meeting the SCI physical activity guidelines (PAG) [32]; were able to read and write English; and had access to a Smartphone. Participants were recruited through advertisements emailed from community organizations from across British Columbia. 9 individuals expressed interest in this study. After screening, six men (aged 27-50 years, $M$ $=39.33 \pm 8.24)$ with $\mathrm{SCI}$ of greater than 1 year duration (6-17 years; $M=12.33$ years), and experiencing belowlevel neuropathic pain were eligible for study participation and were included in this study. Some people with SCI may experience more than one type of pain (e.g., person with $\mathrm{SCI}<\mathrm{C} 7$ who has musculoskeletal shoulder pain, and atlevel neuropathic pain). We deliberately recruited participants who self-identified as experiencing below-level (compared to at-level) neuropathic pain to minimize the risk of pain misidentification and measurement error. UBC Behavioural Research Ethics Board approved this study protocol, and participants provided written informed consent prior to enrolling in the study.

\section{Study design}

This descriptive, observational study employed a case series design.

\section{Equipment mEMA}

mEMA by ilumivu [33] is a Smartphone application designed for use on both Android and i-OS compatible devices. This application was used to deliver prompts to participants to complete the study measures and to collect the study data.

\section{Fitbit surge heart-rate monitors}

Fitbit Surge wrist-worn heart rate (HR) monitors were worn by participants to collect HR data in order to corroborate the timing of self-reported bouts of exercise. These monitors have been tested on persons with SCI against a validated, ActiHeart HR monitor and showed a strong correlation of heart rates between both devices $(r=0.64 ; p<0.001)$ [34]. 
However, the HR variations between the two monitors were larger when HR was $>100 \mathrm{bpm}$ (i.e., during moderate to vigorous physical activity). Exact HR values recorded by FitBit Surges may not necessarily be accurate, therefore beat-by-beat HR data was not recorded. Rather, FitBit Surges were used strictly for identifying large fluctuations in heart rates that would signify exercise participation.

\section{Measures}

\section{Neuropathic pain}

Participants' neuropathic pain was measured using a modified version of the Neuropathic Pain Scale (NPS) [35]. This 10-item scale measures pain qualities typical of neuropathic pain (e.g., 'sharp', 'sensitive', 'hot'), in addition to two general qualities describing pain 'intensity' and overall pain 'unpleasantness' $(0=$ nothing at all, $10=$ most intense sensation imaginable). One question from the NPS was excluded (i.e., 'which of the following best describes the time quality of your pain') because it was not meaningful given the repeated administration of the item over the course of a day. Scores for NPS items were averaged to form a composite pain score for each individual mEMA prompt. The NPS has been validated among people with various neuropathic pain syndromes (including $\mathrm{SCI}$ ) and has been shown to have sensitivity to detect effects of treatment [35].

\section{Affect}

Hardy and Rejeski's 11-point, single item Feeling Scale (FS) and Svebak and Murgatroyd's Felt Arousal Scale (FAS) were used to measure participants' affect and arousal in response to exercise and throughout the day [36, 37]. The 11-point, single item FS measured participants' overall feeling of pleasure-displeasure $(-5=$ very bad, $+5=$ very good), whereas the FAS measured participants' perceived activation $(1=$ low arousal, $6=$ high arousal). Using the FS alongside the FAS enhances construct validity by assessing activation in addition to affective valence (i.e., pleasuredispleasure) [38]. The FS and FAS have not been validated in the SCI population, however previous research suggests the FS is responsive to exercise participation of individuals with SCI [26].

\section{Protocol}

While participating in the study, participants were instructed to maintain their usual activities. At the time of study enrolment, participants were asked which days and times they expected to exercise throughout the 6-day protocol. As per the SCI PAG [32], participants were required to exercise at least 2 times; exercise bout \#1 refers to the first day of exercise within the study, and exercise bout \#2 refers to the second day of exercise within the study. This information allowed the researcher to program mEMA to send prompts both pre and post-exercise, and 4 other times per day. Each participant was contacted the evening prior to the anticipated exercise day to confirm their exercise time. This strategy ensured that the exercise-related mEMA prompts were accurately timed. All other mEMA prompts were randomized within pre-determined intervals. A total of 6 prompts per day, over the course of 6 days were sent to participants. The interval schedule was designed based on previous research involving adults with a physical disability $[18,39]$.

For exercise-related prompts, participants completed the questionnaires immediately before exercise and within $1 \mathrm{~h}$ after exercise. For all other prompts, questionnaires were to be completed within a maximum of $15 \mathrm{~min}$ following the receipt of the mEMA prompt, otherwise, data for that prompt was marked as missing. The order of administration of the neuropathic pain and affect questionnaires was systematically rotated to control for any order of presentation biases.

\section{Statistical analyses}

Data were analyzed at both the group level, and the individual level. At the group level, a paired samples $t$-test was conducted to test whether neuropathic pain and positive affect changed from before to after exercise participation. Independent $t$-tests were conducted for both bouts of exercise, with significance set at $p<.05$.

Following each $t$-test, effect sizes were calculated using Laken's effect size calculator [40]. Cohen's $\mathrm{d}_{a v}$ was calculated; however, it is based on sample estimates and is positively biased, therefore Hedge's $\mathrm{g}_{a v}$ correction was applied [40]. Effect sizes were interpreted according to Cohen's conventions (small $=0.20$, medium $=0.50$, large $=0.80$ ) [41]. Additionally, percentage of change in neuropathic pain from pre-exercise to post-exercise was calculated for each bout, with a 30\% reduction in pain intensity considered to be clinically significant [42].

At the individual level, data were plotted for each participant to examine changes from pre to post-exercise and over the course of each day (Fig. 1a-2b). Bivariate Pearson's correlation coefficients were computed between time of day, neuropathic pain and affect. These analyses were conducted within-participants because calculating correlations across all participants would violate the assumption of independence of observations [43] (i.e., time of day data points are nested within participants). 

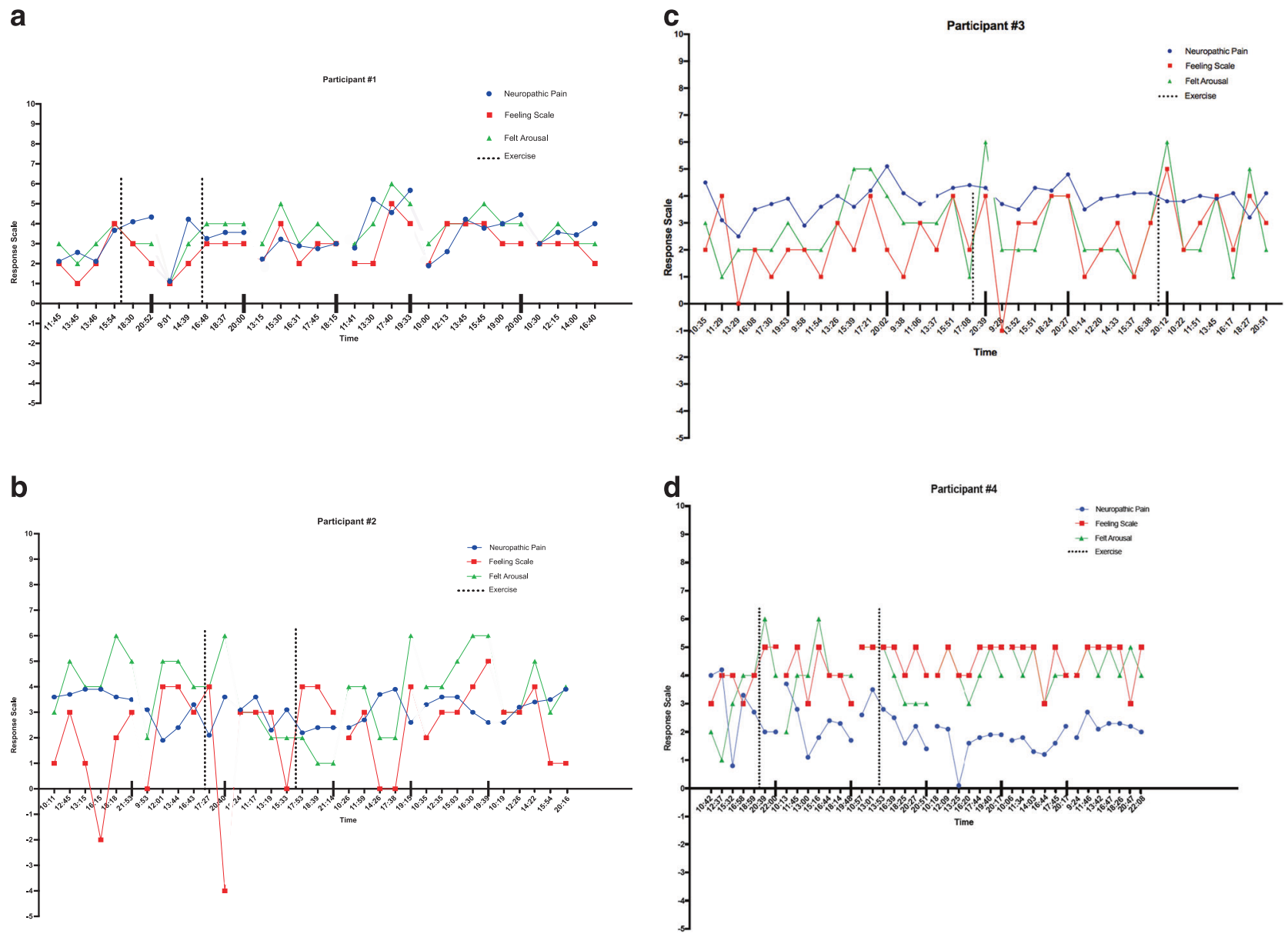

Fig. 1 a Diurnal variations in NP, FS, FA: Participant 1. b Diurnal variations in NP, FS, FA: Participant 2. c Diurnal variations in NP, FS, FA: Participant 3. d Diurnal variations in NP, FS, FA: Participant 4

\section{Results}

\section{Participant compliance}

Across the 6-day study protocol, participant compliance rates for EMA surveys ranged from 80.6-113.9\% $(M=$ 94.9\%). This range was due to technological difficulties associated with mEMA (i.e., failure to send a prompt, or sending multiple prompts within an interval leading to $>100 \%$ compliance), or participants not completing the surveys within the required 15 -min time frame.

\section{Neuropathic pain}

Descriptive statistics and $t$-tests for neuropathic pain are presented in Table 1. For exercise bout \#1, there was a nonsignificant small decrease in NPS scores from pre- to postexercise $\left(\mathrm{Hg}_{a v}=0.18 ;-7.8 \%\right)$. For exercise bout \#2, a significant medium-sized decrease in NPS scores occurred from pre to post-exercise $\left(\operatorname{Hg}_{a v}=0.52 ;-19.9 \% ; p=\right.$ $0.011)$.

\section{Affect}

Descriptive statistics and t-tests for FS and FA scores are presented in Table 1. A small, non-significant increase in FS scores was reported between pre- and post-exercise bout \#1 $\left(\mathrm{Hg}_{a v}=0.22\right)$. However, a non-significant medium to large increase was reported for FS scores from pre- to postexercise bout \#2 $\left(\mathrm{Hg}_{a v}=0.76\right)$. For FA scores, there was no significant difference between pre and post-exercise for bout \#1 or \#2. The effect sizes were very small for both exercise bout \#1 $\left(\mathrm{Hg}_{a v}=0.10\right)$ and bout $\# 2\left(\mathrm{Hg}_{a v}=0.11\right)$.

\section{Intra-individual results}

Participant 1: Following exercise bout \#1, Participant \#1 reported increased neuropathic pain and displeasure, but lower arousal. After exercise bout \#2, he reported decreased neuropathic pain, greater feelings of pleasure, but no change in arousal. Significant positive correlations between NPS and FS scores $(r=0.480, p=0.007)$, and NPS and FA scores $(r=0.610, p<0.001)$ were observed. Time of day 


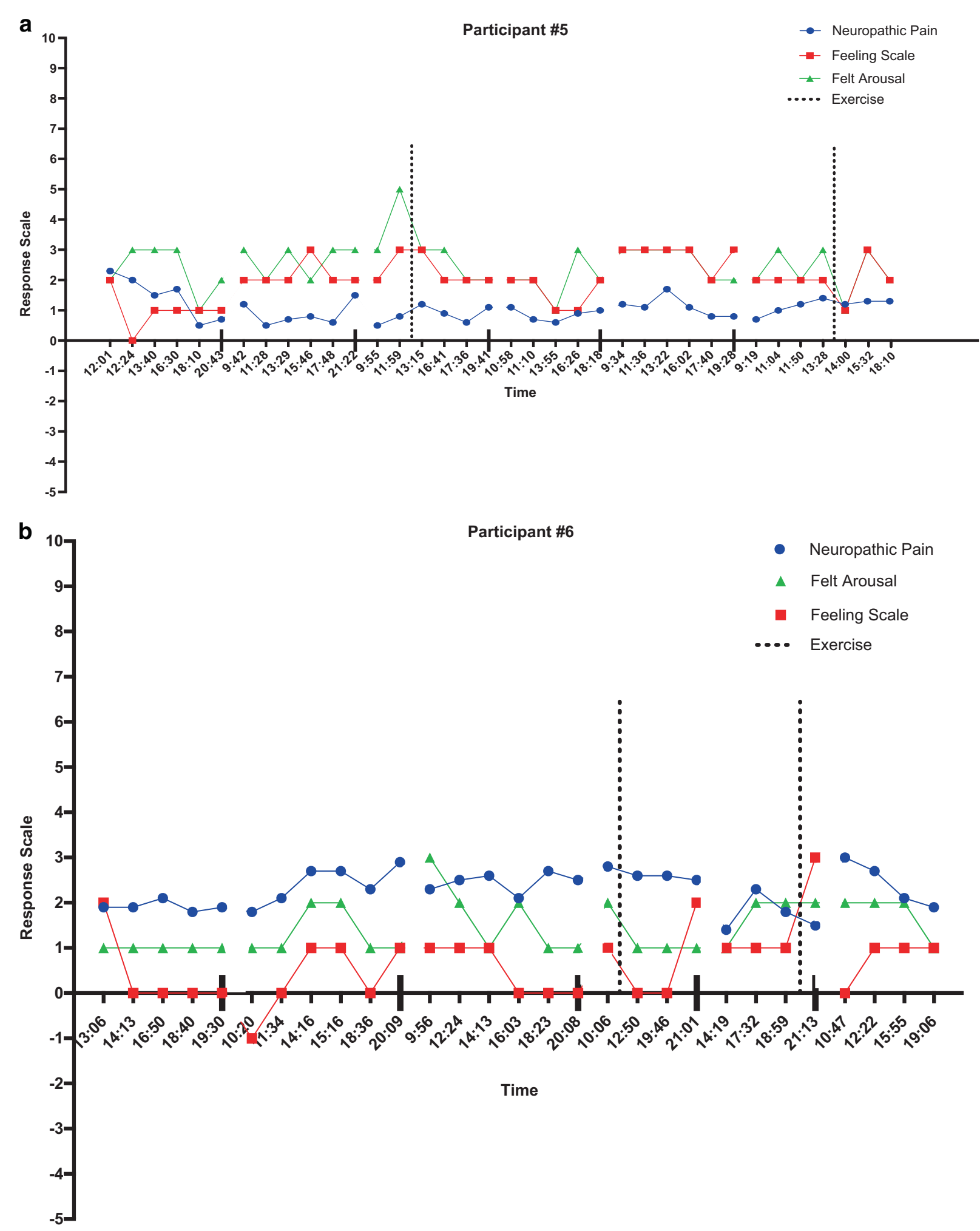

Fig. 2 a Diurnal variations in NP, FS, FA: Participant 5. b Diurnal variations in NP, FS, FA: Participant 6

was significantly positively correlated with NPS ( $r=0.623$, $p<0.001)$, and FA scores $(r=0.449, p=0.013)$, but not FS scores $(r=0.354, p=0.055)$. These results suggest that as neuropathic pain increased, so did arousal. As time of day progressed, so did sensations of neuropathic pain and arousal (Fig. 1a). 
Table 1 Paired samples $t$-tests comparing NPS, FS and FA from pre to post-exercise (Exercise Bout \#1 and Bout \#2)

\begin{tabular}{|c|c|c|c|c|c|c|c|}
\hline \multirow[t]{2}{*}{ Pair } & \multirow[t]{2}{*}{$N$} & \multirow[t]{2}{*}{ Mean } & \multirow[t]{2}{*}{ Standard deviation } & \multirow[t]{2}{*}{$t$} & \multirow[t]{2}{*}{ Sig. } & \multicolumn{2}{|c|}{$\begin{array}{l}\text { 95\% Confidence } \\
\text { interval }\end{array}$} \\
\hline & & & & & & Lower & Upper \\
\hline \multicolumn{8}{|l|}{ Neuropathic pain } \\
\hline Pre exercise Bout \#1 NP & 6 & 2.95 & 1.22 & 0.882 & 0.418 & -0.437 & 0.894 \\
\hline Post exercise Bout \#1 NP & & 2.72 & 1.24 & & & & \\
\hline Pre exercise Bout \#2 NP & 6 & 3.02 & 1.18 & 3.93 & 0.011 & 0.212 & 1.01 \\
\hline Post exercise Bout \#2 NP & & 2.41 & 1.00 & & & & \\
\hline Feeling scale & & & & & & -1.60 & 0.938 \\
\hline Pre exercise Bout \#1 FS & 6 & 2.833 & 1.17 & -0.674 & 0.530 & & \\
\hline Post exercise Bout \#1 FS & & 3.17 & 1.72 & & & & \\
\hline Pre exercise Bout \#2 FS & 6 & 2.17 & 1.72 & -1.87 & 0.121 & -3.17 & 0.504 \\
\hline Post exercise Bout \#2 FS & & 3.50 & 1.52 & & & & \\
\hline Felt arousal & & & & & & -2.77 & 2.44 \\
\hline Pre exercise Bout \#1 FA & 6 & 3.33 & 1.51 & -0.164 & 0.876 & & \\
\hline Post exercise Bout \#1 FA & & 3.50 & 1.64 & & & & \\
\hline Pre exercise Bout \#2 FA & 6 & 3.00 & 1.10 & -0.237 & 0.822 & -1.97 & 1.64 \\
\hline Post exercise Bout \#2 FA & & 3.17 & 1.83 & & & & \\
\hline
\end{tabular}

Participant 2: Following both exercise bouts \#1 and \#2, participant \#2 experienced a decrease in neuropathic pain and increased feelings of pleasure but no changes in arousal. NPS was significantly, negatively correlated with FS scores ( $r=-0.580, p<0.001)$, but not FA scores $(r=0.121, p=$ $0.495)$. Time of day was not significantly correlated with NPS $(r=0.019, p=0.915)$, FS scores $(r=-0.047, p=$ $0.793)$, or FA scores $(r=0.180, p=0.309)$. These results suggest that as neuropathic pain increased, the participant's feelings of displeasure increased but arousal did not. Furthermore, time of day was not related to neuropathic pain, feelings of pleasure/displeasure, or arousal for this participant (Fig. 1b).

Participant 3: Participant \#3 reported a slight decrease in neuropathic pain and a large increase in overall affect following exercise bout \#1. He experienced a slight decrease in neuropathic pain, and increased feelings of pleasure and arousal following exercise bout \#2. NPS was not significantly correlated with FS scores $(r=0.255, p=0.139)$ or FA scores $(r=0.241, p=0.163)$. Time of day, however, was significantly positively correlated with NPS $(r=0.352$, $p=0.038)$, FS scores $(r=0.507, p=0.002)$, and FA scores $(r=0.463, p=0.005)$. These results suggest that neuropathic pain, feelings of pleasure, and arousal of participant \#3 increased as time of day progressed (Fig. 1c).

Participant 4: Following exercise bout \#1, participant \#4 experienced decreased neuropathic pain and increased feelings of pleasure and arousal. Exercise bout \#2 led to decreased neuropathic pain and arousal, with no change in feelings of pleasure/displeasure. NPS was not significantly correlated with FS scores $(r=-0.009, p=0.955)$ or FA scores $(r=-0.251, p=0.114)$. Furthermore, time of day was not significantly correlated with NPS $(r=-0.242, p=$ $0.127)$, FS scores $(r=-0.008, p=0.959)$, or FA scores $(r$ $=0.072, p=0.653)$. These results suggest that neuropathic pain, affect, and time of day were not related for participant \#4 (Fig. 1d).

Participant 5: Following exercise bout \#1, participant \#5 reported an increase in neuropathic pain, decreased arousal but no changes in feelings of pleasure/displeasure. He reported decreased neuropathic pain, greater displeasure and decreased arousal following exercise bout \#2. NPS was not significantly correlated with FS scores $(r=-0.097, p=$ $0.572)$, or FA scores $(r=0.216, p=0.206)$. No significant correlations were found between time of day and NPS ( $r=$ $-0.094, p=0.596)$, FS scores $(r=-0.129, p=0.453)$, or FA scores $(r=-0.220, p=0.197)$ (Fig. 2a).

Participant 6: Participant \#6 experienced a decrease in neuropathic pain following both exercise bouts. Following exercise bout \#1, he experienced a slight increase in displeasure and a slightly decreased state of arousal. He experienced increased feelings of pleasure with no changes in arousal following exercise bout \#2. NPS was not significantly correlated with FS scores $(r=-0.107, p=0.581)$ or FA scores $(r=0.177, p=0.358)$. Time of day was not significantly correlated with NPS $(r=-0.244, p=0.202)$, FS scores $(r=0.188, p=0.329)$, or FA scores $(r=-0.332$, $p=0.079$ ) (Fig. 2b).

\section{Discussion}

To the best of our knowledge, this is the first SCI study to evaluate the acute effects of exercise on both neuropathic 
pain and affective states within a real-world setting, and to report on diurnal variations in neuropathic pain and affect. Consistent with our hypotheses, following at least one bout of exercise, all study participants experienced a neuropathic pain reduction, and 5 out of 6 participants experienced an increase in affect. These results align with research on general body pain in adults with SCI [12-15, 26] and support exercise as a potential method to reduce neuropathic pain and improve affect. Furthermore, our study showed that some participants ( 2 out of 6 ) experienced an increase in their neuropathic pain and arousal as the day progressed.

Although all six participants experienced a decrease in neuropathic pain following at least one exercise bout, two participants experienced an increase in pain after one exercise bout. These idiosyncrasies might be attributable to the participants' choice of exercise intensity. In the exercise training study conducted by Norrbrink and colleagues [16], participants exercised at $70-100 \%$ of their maximum HR (above the previously suggested HR max of 50-80\%) [32] which supports the idea that exercise may need to be of a higher intensity to induce improvements in pain. Norrbrink et al.'s finding aligns with research showing that in order to derive some physiological benefits of exercise (e.g., improvements in HR variability), adults with SCI need to have an average exercising HR of greater than 100 beats per minute [44-46]. It is possible that 2 participants did not experience a decrease in their neuropathic pain following one bout of exercise because they were not exercising at a sufficient intensity. Determining how the intensity of exercise participation may influence individual neuropathic pain responses to exercise training is an important area of research to target.

Results at the group level showed that feelings of pleasure increased from pre-exercise to post-exercise bout \#2, however this increase was not statistically significant. Although research is very limited regarding the interrelationships between exercise, feeling states, and pain experienced by individuals with SCI, one study found that acute exercise-related changes in pain were significantly, negatively correlated with changes in feeling states [26]. Results from the current study support these findings insofar as the exercise bout with the largest increase in affect was also the bout with significant decreases in pain. Therefore, it may have been the decrease in neuropathic pain reported by study participants that led to the improvement in their feeling states. As with neuropathic pain, the intensity of exercise required to stimulate optimal affective responses remains highly debated [47].

Although some significant relationships were observed between time of day, neuropathic pain, and affect, heterogeneous results were observed across participants. To the best of our knowledge, only one other study has examined momentary aspects of pain in the SCI population [18].
Results showed that chronic pain steadily increased from wake until 7 PM, and then slightly decreased before bedtime. One reason for neuropathic pain worsening (for some participants) as the time of day progresses may be due to the reduction of mental stimulation at nighttime. More distractions are present during the day for the majority of individuals, which may inhibit neuropathic pain from being the most prominent thought in the minds of those who experience it. Although two participants in our study reported a similar pattern to that reported by Kratz and colleagues [18], our results better align with a case series which showed that some individuals exhibited a temporal pattern in their pain intensities, but others did not [48]. Overall, results from this study support general findings that diurnal patterns exist between time of day, neuropathic pain and affect, but these relationships are inconsistent across individuals.

\section{Study strengths and limitations}

This study has several strengths. First, there was very little missing data and no participant dropout. We attribute this strength to pilot-testing the study protocol, as it indicated that this measurement schedule was not cumbersome, and therefore minimized the probability of reduced data quality and quantity [49]. Second, the utility of using a mobile EMA platform to observe diurnal variations in pain and affect was demonstrated. Therefore, this study provides a methodology for future EMA studies in the SCI population. Repeated, electronic assessments may be useful for observing temporal changes between psychosocial, nutritional, and pain-related constructs. Third, the ecological nature of this case series allowed for a deeper understanding of intraindividual differences in neuropathic pain and affect, both in response to exercise and over the course of each day. Relationships observed between these constructs provide rationale to further examine potential variables that may explain these patterns, such as the intensity of exercise participation.

Despite these strengths, some limitations must be noted. First, participants' neuropathic pain may have been exacerbated due to repeatedly asking them to think about their pain levels. However, it was anticipated that heightened neuropathic pain due to increased awareness would remain consistent across the measurement period. This limitation was noted in the study consent form sent to participants, however only 2 participants mentioned an increase in their pain resulting from repeated assessments. Second, given the naturalistic nature of this study, exercise frequency, intensity, and type were not experimentally controlled. However, the observed reductions in neuropathic pain and increase in positive affect following such varied exercise bouts supports the notion that exercise may be a robust potential 
neuropathic pain management option for some adults with SCI. Third, the International Data Sets have been introduced to measure neuropathic pain in adults with SCI [50]. However, this study used the NPS due to its' brevity, and ability to assess responses to treatment [35].

\section{Conclusion}

Taken together, the results of this study suggest that for some people with SCI, exercise may be used to reduce neuropathic pain, and to simultaneously enhance feelings of pleasure. Moreover, for some individuals, neuropathic pain and affect may vary as a function of time of day. Using a momentary assessment tool may help identify individual diurnal pain patterns and responses to exercise. Future research should be directed towards understanding the conditions under which exercise may be used as either an alternative to, or in conjunction with, pharmacotherapy to acutely reduce neuropathic pain in adults with SCI.

Authors contributions KMG supervised the project and provided assistance with design of the research study, measurement selection, obtaining ethics approval, analysis and interpretation of the data, and manuscript preparation. KT was responsible for authoring the ethics application that was submitted to the BREB at UBC (Okanagan), as well as the design of the research study. Furthermore, she selected which measures would be used, and was the sole investigator responsible for participant recruitment, participant contact and training participants how to utilize the apparatus. Last, KT was responsible for all data collection, input and analysis.

\section{Compliance with ethical standards}

Conflict of interest The authors declare that they have no conflict of interest.

\section{References}

1. Finnerup NB, Jensen MP, Norrbrink C, Trok K, Johannesen IL, Jensen TS, et al. A prospective study of pain and psychological functioning following traumatic spinal cord injury. Spinal Cord. 2016;54:816-21.

2. Burke D, Fullen BM, Stokes D, Lennon O. Neuropathic pain prevalence following spinal cord injury: A systematic review and meta-analysis. Eur J Pain. 2017;21:29-44.

3. IASP. IASP taxonomy. 2012. . http://www.iasp-pain.org/Educa tion/Content.aspx $?$ ItemNumber $=1698 \&$ navItemNumber $=576$. Accessed Date April 4, 2018

4. Anson CA, Shepherd C. Incidence of secondary complications in spinal cord injury. International journal of rehabilitation research. Internationale Zeitschrift fur Rehabilitationsforschung. Rev Int De Rech De Readapt. 1995;19:55-66.

5. Backonja MM, Stacey B. Neuropathic pain symptoms relative to overall pain rating. J Pain. 2004;5:491-7.

6. Cruz-Almeida Y, Felix ER, Martinez-Arizala A, WiderströmNoga EG. Pain symptom profiles in persons with spinal cord injury. Pain Med. 2009;10:1246-59.
7. Jensen TS, Finnerup NB. Allodynia and hyperalgesia in neuropathic pain: clinical manifestations and mechanisms. Lancet Neurol. 2014;13:924-35.

8. Guy SD, Mehta S, Casalino A, Côté I, Kras-Dupuis A, Moulin $\mathrm{DE}$, et al. The CanPain SCI clinical practice guidelines for rehabilitation management of neuropathic pain after spinal cord: recommendations for treatment. Spinal Cord. 2016;54(S1):S14.

9. Ragnarsson K. Management of pain in persons with spinal cord injury. J Spinal Cord Med. 1997;20:187-99.

10. Warms CA, Turner JA, Marshall HM, Cardenas DD. Treatments for chronic pain associated with spinal cord injuries: many are tried, few are helpful. Clin J Pain. 2002;18:154-63.

11. Hutchinson KJ, Gómez-Pinilla F, Crowe MJ, Ying Z, Basso DM. Three exercise paradigms differentially improve sensory recovery after spinal cord contusion in rats. Brain. 2004;127:1403-14.

12. Boldt I, Eriks-Hoogland I, Brinkhof MW, de Bie R, Joggi D, von Elm E. Non-pharmacological interventions for chronic pain in people with spinal cord injury. Cochrane Database Syst Rev. 2014;11:69-74.

13. Crane DA, Hoffman JM, Reyes MR. Benefits of an exercise wellness program after spinal cord injury. J Spinal Cord Med. 2015;25:1-5.

14. Hicks AL, Martin KA, Ditor DS, Latimer AE, Craven C, Bugaresti $\mathrm{J}$, et al. Long-term exercise training in persons with spinal cord injury: effects on strength, arm ergometry performance and psychological well-being. Spinal Cord. 2003;41:34-43.

15. Martin Ginis KA, Latimer AE, McKecknie K, Ditor DS, McCartney N, Hicks AL. Using exercise to enhance subjective wellbeing among people with spinal cord injury: the mediating influences of stress and pain. Rehabil Psychol. 2003;48:157-64.

16. Norrbrink C, Lindberg T, Wahman K, Bjerkefors A. Effects of an exercise programme on musculoskeletal and neuropathic pain after spinal cord injury-results from a seated double-poling ergometer study. Spinal Cord. 2012;50:457-61.

17. Celik EC, Erhan B, Lakse E. The clinical characteristics of neuropathic pain in patients with spinal cord injury. Spinal Cord. 2012;50:585-9.

18. Kratz AL, Ehde DM, Bombardier CH, Kalpakjian CZ, Hanks RA. Pain acceptance decouples the momentary associations between pain, pain interference, and physical activity in the daily lives of people with chronic pain and spinal cord injury. J Pain. 2017;18:319-31.

19. Shiffman S, Stone AA, Hufford MR. Ecological momentary assessment. Annu Rev Clin Psychol. 2008;4:1-32.

20. May M, Junghaenel DU, Ono M, Stone AA, Schneider S. Ecological momentary assessment methodology in chronic pain research: a systematic review. J Pain. 2018;19:699-716.

21. Ekkekakis P. The measurement of affect, mood, and emotion: a guide for health-behavioral research. Cambridge; Cambridge University Press; 2013.

22. Rodrigues D, Tran Y, Wijesuriya N, Guest R, Middleton J, Craig A. Pain intensity and its association with negative mood States in patients with spinal cord injury. Pain Ther. 2013;2:113-9.

23. Hagglund KJ, Haley WE, Reveille JD, Alarcon GS. Predicting individual differences in pain and functional impairment among patients with rheumatoid arthritis. Arthritis Rheum. 1989;32:851-8.

24. Smedstad LM, Mourn T, Vaglum P, Kvien TK. The impact of early rheumatoid arthritis on psychological distress. Scand J Rheumatol. 1996;25:377-82.

25. Dua JK. The role of negative affect and positive affect in stress, depression, self-esteem, assertiveness, type A behaviors, psychological health, and physical health. J Soc Psychol. 1993;119:515-52. 
26. Ginis KM, Latimer AE. The effects of single bouts of bodyweight supported treadmill training on the feeling states of people with spinal cord injury. Spinal Cord. 2007;45:112-5.

27. Bani MA, Arazpour M, Farahmand F, Kashani RV, Mousavi ME, Hutchins SW. Comparison of new medial linkage reciprocating gait orthosis and isocentric reciprocating gait orthosis on energy consumption in paraplegic patients: a case series. Spinal Cord Ser Cases. 2015;1:15012.

28. Grassner L, Geuther M, Mach O, Bühren V, Vastmans J, Maier D. Charcot spinal arthropathy: an increasing long-term sequel after spinal cord injury with no straightforward management. Spinal Cord Ser Cases. 2015;1:15022.

29. Jayaraman A, Shah P, Gregory C, Bowden M, Stevens J, Bishop $\mathrm{M}$, et al. Locomotor training and muscle function after incomplete spinal cord injury: case series. J Spinal Cord Med. 2008;31:185-93.

30. Kumru H, Albu S, Vidal J, Barrio M, Santamaria J. Dopaminergic treatment of restless legs syndrome in spinal cord injury patients with neuropathic pain. Spinal Cord Ser Cases. 2016;2:16022.

31. Pandey S, Holla VV, Rizvi I, Qavi A, Shukla R. Can vitamin B12 deficiency manifest with acute posterolateral or posterior cord syndrome? Spinal Cord Ser Cases. 2016;2:16006.

32. Ginis KM, Hicks AL, Latimer AE, Warburton DE, Bourne C, Ditor DS, et al. The development of evidence-informed physical activity guidelines for adults with spinal cord injury. Spinal Cord. 2011;49:1088-96.

33. Tuomenoksa, M. Ilumivu: ecological momentary assessment. 2013. http://www.ilumivu.com/index.php?section=41\&chapter $=$ 54. Accessed Date April 4, 2018

34. Tsang KL, Yong HJR, Ding D. Measuring heart rate in manual wheelchair users during exercise and free-living activity with the latest Fitbit surge monitor. conference: rehabilitation engineering and assistive technology society of North America. 2016.

35. Galer BS, Jensen MP. Development and preliminary validation of a pain measure specific to neuropathic pain The Neuropathic Pain Scale. Neurology. 1997;48:332-8.

36. Hardy CJ, Rejeski WJ. Not what, but how one feels: the measurement of affect during exercise. J Sport Exerc Psychol. 1989;11:304-17.

37. Svebak S, Murgatroyd S. Metamotivational dominance: a multimethod validation of reversal theory constructs. J Pers Soc Psychol. 1985;48:107-16.
38. Watson D, Clark LA. Measurement and mismeasurement of mood: recurrent and emergent issues. J Pers Assess. 1997;8:267-96.

39. Focht BC, Ewing V, Gauvin L, Rejeski WJ. The unique and transient impact of acute exercise on pain perception in older, overweight, or obese adults with knee osteoarthritis. Ann Behav Med. 2002;24:201-10.

40. Lakens D. Calculating and reporting effect sizes to facilitate cumulative science: a practical primer for $t$-tests and ANOVAs. Front Psychol. 2013;4:863.

41. Cohen J. Statistical power analysis for the behavioural sciences (Revised edition). Academic Press; New York;1989. p. 7.

42. Farrar JT, Young Jr JP, LaMoreaux L, Werth JL, Poole RM. Clinical importance of changes in chronic pain intensity measured on an 11-point numerical pain rating scale. Pain. 2001;94:149-58.

43. Field A. Discovering statistics using IBM SPSS statistics. Cambridge;Sage; 2013.

44. Ditor DS, Kamath MV, MacDonald MJ, Bugaresti J, McCartney N, Hicks AL. Effects of body weight-supported treadmill training on heart rate variability and blood pressure variability in individuals with spinal cord injury. J Appl Physiol. 2005;98:1519-25.

45. Grigorean VT, Sandu AM, Popescu M, Iacobini MA, Stoian R, Neascu C, et al. Cardiac dysfunctions following spinal cord injury. J Med Life. 2009;2:133.

46. Elkington TJ, Cassar S, Nelson AR, Levinger I. Psychological responses to acute aerobic, resistance, or combined exercise in healthy and overweight individuals: a systematic review. Clin Med Insights Cardiol. 2017;11:1-23.

47. Ekkekakis P, Hall EE, Petruzzello SJ. Variation and homogeneity in affective responses to physical activity of varying intensities: an alternative perspective on dose-response based on evolutionary considerations. J Sports Sci Med. 2005;23:477-500.

48. Strian F, Lautenbacher S, Galfe G, Hölzl R. Diurnal variations in pain perception and thermal sensitivity. Pain. 1989;36:125-31.

49. Rolstad S, Adler J, Rydén A. Response burden and questionnaire length: is shorter better? A review and meta-analysis. Value Health. 2011;14:1101-8.

50. Widerström-Noga E, Biering-Sørensen F, Bryce TN, Cardenas DD, Finnerup NB, Jensen MP, et al. The international spinal cord injury pain basic data set (version 2.0). Spinal Cord. 2014;52:282-6. 